\title{
Fístulas coronario-pulmonares asintomáticas. Reporte caso
}

\author{
Asymptomatic coronary-pulmonary fistulas. Case report \\ Fístulas coronário-pulmonares assintomáticaso. Relato de caso
}

Jenny Carolina Salazar F. ORCID: 0000-0002-1040-8929 Médico general. Departamento de urgencias. Hospital Universitario de Santander. Bucaramanga, Colombia.

Diego Alejandro Rangel R. ORCID: 0000-0002-4923-1438

Médico. Residente de medicina

Interna. Universidad Industrial

de Santander. Departamento de

Medicina Interna. Grupo GERMINA. Bucaramanga, Colombia.

Néstor Eduardo Ospino R.

ORCID: 0000-0002-5773-0157

Cardiólogo. Especialista en hemodinamia. Departamento de Radiología. Hospital Universitario de Santander. Bucaramanga, Colombia.

Juan Manuel Quirós

ORCID: 0000-0002-2268-7339

Cardiólogo. Especialista en hemodinamia. Departamento de Radiología. Hospital Universitario de Santander. Bucaramanga, Colombia.
Resumen: La fístula coronaria es una anomalía caracterizada por una comunicación anormal entre una arteria coronaria y una cámara cardíaca o un gran vaso. El 50\% de las fístulas se originan de la arteria coronaria derecha, $45 \%$ de la arteria coronaria izquierda y menos del $5 \%$ en ambas. Se presenta una paciente femenina de 81 años de edad con historia de ataque cerebrovascular isquémico de forma previa, quien ingresa con nuevo evento isquémico cerebral. En estudios complementarios se documentó una fracción de eyección del ventrículo izquierdo reducida por lo que se realizó arteriografía con hallazgo de arterias coronarias epicárdicas sin enfermedad obstructiva significativa, pero con fístula coronario-pulmonar en segmento medio de arteria descendente anterior y una fístula coronario-pulmonar en segmento proximal de la arteria coronaria derecha. Las fístulas coronarias son una entidad rara, con síntomas inespecíficos. La mayoría de las veces se diagnostica de forma incidental al realizar una arteriografía coronaria por otro motivo. El manejo varía de acuerdo a las manifestaciones y anatomía encontrada junto con la experiencia del equipo de cardiología.

Palabras Claves: fístula de arteria coronaria, angiografía por tomografía computarizada, angiografía coronaria.

Abstract: Coronary fistula is an abnormality characterized by abnormal communication between a coronary artery and a cardiac chamber or large vessel. Half of fistulas originate from the right coronary artery, $45 \%$ from the left coronary artery, and less than $5 \%$ originate from both. We present an 81-year-old female patient with a history of previous ischemic stroke, who was admitted with a new cerebral ischemic event. In complementary studies, a reduced ejection fraction of the left ventricle was documented, then arteriography was performed showing epicardial coronary arteries without significant obstructive disease, but with coronary-pulmonary fistula in the middle segment of the anterior descending artery and a coronary-pulmonary fistula in the proximal segment of the right coronary artery. Coronary fistulas are a rare entity, with nonspecific symptoms. Most of the time this entity is diagnosed incidentally when performing a coronary arteriography for another reason. Management varies according to the manifestations and anatomy found along with the experience of the cardiology team.

Key words: coronary artery fistula, computed tomography angiography, coronary angiography.

Resumo: A fístula coronária é uma anormalidade caracterizada pela comunicação anormal entre uma artéria coronária e uma câmara cardíaca ou um vaso grande. Metade das fístulas são originárias da artéria coronária direita, $45 \%$ da artéria coronária esquerda e menos de $5 \%$ são originárias de ambas. Se você apresentar um paciente feminino de 81 anos de idade com história de ataque cerebrovascular isquêmico de forma prévia, que ingressou com o novo evento isquêmico cerebral. Em estudos complementares, documentou-se uma fração de ejeção reduzida do ventrículo esquerdo e, em seguida, foi realizada arteriografia mostrando artérias coronárias epicárdicas sem doença obstrutiva, mas com uma fístula coronário-pulmonar no segmento médio da artéria descendente anterior e uma fístula coronário-pulmonar no segmento proximal da artéria artéria coronária direita. As fístulas coronárias são uma entidade rara, com sintomas inespecíficos. Na maioria das vezes, essa entidade é diagnosticada incidentalmente 
ao realizar uma arteriografia coronariana por outro motivo. O manejo varia de acordo com as manifestações e anatomia encontradas, juntamente com a experiência da equipe de cardiologia.

Palavras-chave: fístula arterial coronariana, angiotomografia computadorizada, angiografia coronariana. 


\section{Introducción}

Las fístulas coronarias fueron descritas inicialmente por Krause en el año 1865 y consisten en una circulación coronaria anómala dado por una comunicación entre una arteria coronaria con una estructura adyacente ${ }^{(1)}$. Corresponde al 1-4\% de las cardiopatías congénitas, aunque también puede ser adquirida, secundaria a procedimientos invasivo o trauma torácico (2). Asimismo, entre el 5-30\% de las mismas se encuentran asociadas a una anomalía congénita ${ }^{(3)}$.

Se describe una incidencia entre $0,1-0,2 \%$ en la población adulta sometida a angiografía coronaria, con una relación hombre-mujer de 1,9:1 (4). Aproximadamente el $50 \%$ de las fístulas se originan de la arteria coronaria derecha (ACD), $45 \%$ de la coronaria izquierda y menos del $5 \%$ se originan de ambas. De acuerdo al calibre, tamaño y conexión se modifica la presentación clínica. En general, la mayoría de las fístulas coronarias son asintomáticas y las manifestaciones suelen aparecer cuando el tamaño de la fístula es el doble del calibre de la arteria coronaria (5). Los síntomas más frecuentes incluyen el dolor torácico y disnea ${ }^{(5-7)}$.

Se presenta el caso clínico de una paciente quien en estudio etiológico de cardiopatía con fracción de eyección reducida (estadio B de acuerdo a la clasificación de la American Heart Association) se comprueba la presencia de dos fístulas coronarias -una desde arteria descendente anterior (ADA) y otra desde ACD hacia circulación pulmonar.

\section{Caso clínico}

Paciente femenina de 81 años de edad quien ingresó al servicio de urgencias con cuadro clínico compatible con crisis epiléptica de novo. Antecedentes de hipertensión arterial crónica con mala adherencia al tratamiento, eventos de ataque cerebrovascular isquémico en dos ocasiones (dos y ocho años previos), hipotiroidismo primario, demencia vascular moderada y exposición a combustión de biomasa. No refería historia de disnea, dolor torácico, ortopnea o edema de extremidades.

Al examen físico presentaba apirética, frecuencia cardíaca de $88 \mathrm{cpm}$, frecuencia respiratoria de $18 \mathrm{rpm}$ y saturación de 95\% (FiO2 al 21\%). El estado de conciencia era alerta, desorientada en tiempo, bradipsiquica. El resto del examen neurológico era normal. Al examen cardíaco presentaba un ritmo regular de $88 \mathrm{cpm}$, los ruidos cardíacos eran normales y los silencios libres. A la auscultación pleuropulmonar no presentaba estertores.

La tomografía de cráneo simple mostró cambios crónicos de isquemia cerebral junto con hipodensidad en región temporal basal izquierda correspondiente a un infarto en fase subaguda.

Como estudios de su cardiopatía se realiza:

- electrocardiograma mostraba un ritmo sinusal, con frecuencia cardíaca de 75 latidos por minuto con eje - 60, con bloqueo fascicular anterosuperior izquierdo, sin signos de isquemia o sobrecarga de cavidades.

- ecocardiograma transtorácico que evidenció una cardiopatía hipertrófica y deterioro severo de la fracción de eyección del ventrículo izquierdo (FEVI) de 30\%, aquinesia del casquete apical, presión sistólica de ventrículo derecho de $40 \mathrm{~mm} \mathrm{Hg}$.

- anticuerpos IgG para T. cruzi positivos en dos ocasiones por técnica diferente.

- arteriografía coronaria que evidencia tronco coronario común corto sin lesiones, ADA de mediano calibre y largo recorrido hasta ápex sin lesiones significativas. Se visualizó una fistula coronario-pulmonar en segmento medio de ADA (figura 1). La ACD de buen calibre sin lesiones, observando una fístula hacia arteria pulmonar en su segmento proximal (figura 2). En ninguno de los dos casos, se encontró alteración del flujo coronario de forma distal. La arteria circunfleja de buen calibre, sin lesiones. Dichos hallazgos posiblemente sean de origen congénito sin repercusión hemodinámica y que no estaban en relación a la sintomatología de paciente. 
Figura 1: A) Arteriografía a ADA y circunfleja. Se visualiza el inicio (flecha) de la fístula desde segmento medio de ADA. B) Se visualiza porción final de la fístula (flecha) y contraste en arteria pulmonar (círculo rojo).

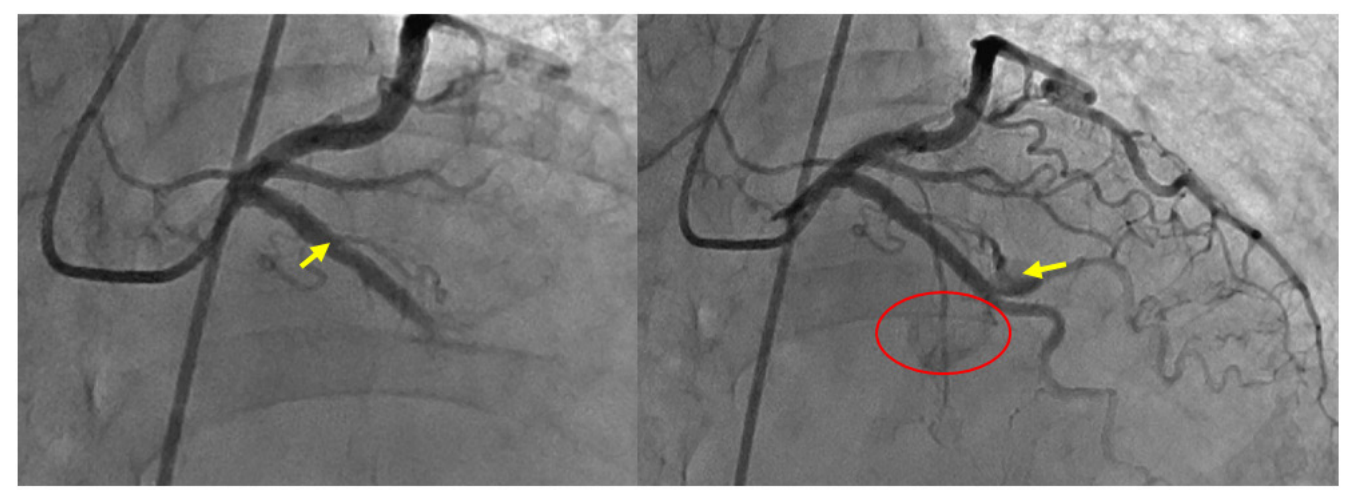

Figura 2: A) Arteriografía selectiva a ACD. Se visualiza el inicio (flecha) y porción final (cabeza de flecha) de la fístula desde segmento proximal de ACD. B) Se visualiza contraste en arteria pulmonar (círculo rojo).

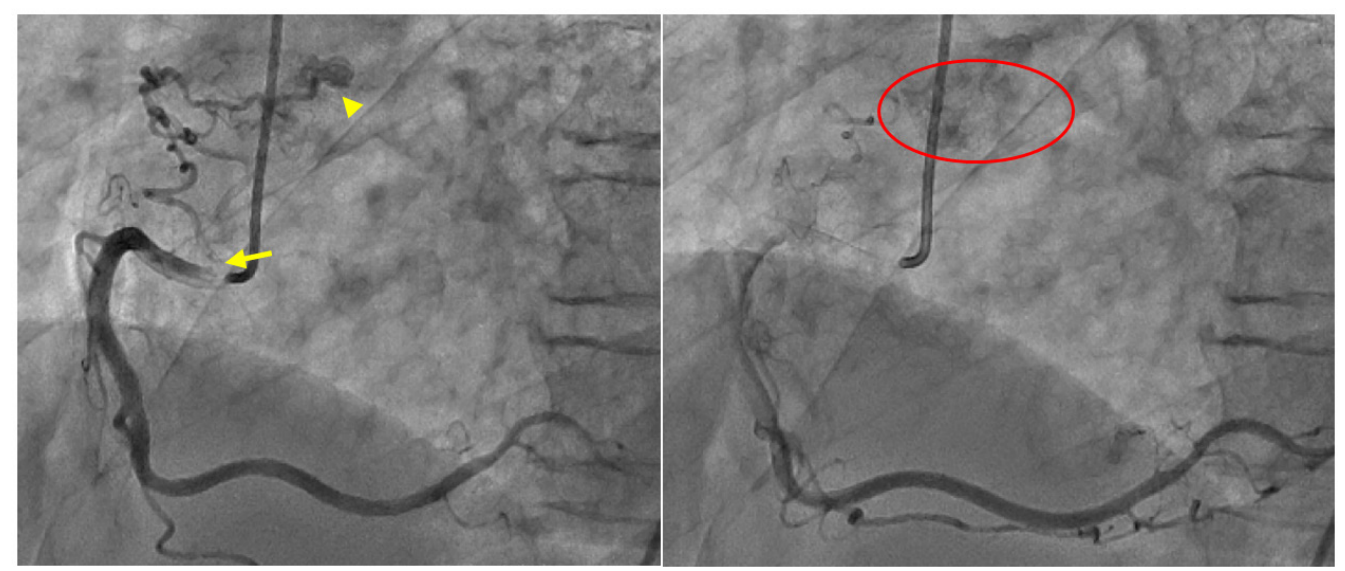

Al séptimo día de hospitalización, se da egreso con tratamiento para cardiopatía con FEVI reducida de origen chagásico y prevención secundaria de evento cerebrovascular.

\section{Discusión}

Las fístulas coronarias pueden tener diferentes comunicaciones a estructuras como una cámara cardíaca, la arteria pulmonar, seno coronario, vena cava o venas pulmonares ${ }^{(8)}$. El $92 \%$ de las fístulas drenan al corazón derecho, y el $8 \%$ al corazón izquierdo - ventrículo derecho (40\%), aurícula derecha (25\%), tronco de arteria pulmonar (15\%), seno coronario (7\%), aurícula izquierda (5\%) y ventrículo izquierdo (menos del $3 \%)^{(4)}$.

La mayoría son de origen congénito, encontrándose de forma aislada o asociada a otras cardiopatías, especialmente con trastornos que afectan el tracto de salida de los ventrículos tales como la atresia pulmonar con tabique interventricular intacto o la atresia aórtica con síndrome de corazón izquierdo hipoplásico ${ }^{(9)}$. De forma más rara, pueden aparecer en casos de trauma (herida torácica por arma corto-punzante o arma de fuego) o iatrogénicas, derivadas de algún procedimiento cardíaco invasivo (implante de marcapasos, cirugía cardíaca, biopsia endomiocárdica o angiografía coronaria) (2).

Los síntomas serán directamente proporcionales al calibre, tamaño y efecto en la circulación coronaria ${ }^{(10,11)}$.

En un estudio reportado por Savioli (10) de 11 casos de fístulas coronarias congénitas, el síntoma más frecuente en 8 pacientes fue dolor torácico, mientras que la disnea de esfuerzo estuvo presente en 2 pacientes. Otros hallazgos reportados incluyen soplo superficial y continuo en el borde paraesternal. Menos frecuentes se observa ortopnea, disnea paroxística nocturna y edema en miembros inferiores ${ }^{(1,7)}$. Se calcula que alrededor del $50 \%$ de los pacientes son asintomáticos o presentan síntomas mínimos ${ }^{(10)}$.

Las fístulas coronarias grandes no tratadas pueden convertirse en sintomáticas y asociarse a secuelas importantes en aproximadamente $19 \%$ de los pacientes menores de 20 años, y $63 \%$ de los pacientes mayores de 20 años ${ }^{(12)}$.

En este sentido, las fístulas pueden favorecer el paso del flujo coronario hacia la circulación pulmonar de baja presión favoreciendo el desarrollo de hipertensión pulmonar o hacia las 
cavidades izquierdas con sobrecarga de dichas cámaras ${ }^{(13)}$. También, se puede producir un fenómeno de robo coronario al redirigir el flujo hacia circulación pulmonar generando isquemia del miocardio distal a la fístula, con dilatación progresiva de la arteria coronaria en su segmento proximal como mecanismo compensatorio ${ }^{(14-16)}$. Otras complicaciones a largo plazo incluyen arritmias, falla cardíaca, endocarditis infecciosa y trombosis ${ }^{(17)}$.

En nuestro caso, dado el tamaño y la ausencia de modificación del flujo coronario de manera distal y consideramos una fístula coronaria de origen congénito sin identificación de clínica asociada.

Dentro del abordaje diagnóstico, es necesario resaltar que la radiografía de tórax y electrocardiograma son generalmente normales, a menos que exista alguna de las complicaciones antes mencionadas.

El ecocardiograma transtorácico tiene bajo rendimiento. En un estudio que incluyó 18 pacientes con fístulas coronarias, sólo 6 tuvieron hallazgos sugestivos de la patología. La sensibilidad mejora al usar el ecocardiograma transesofágico, aunque tiene dificultad para identificarse el sitio de conexión ${ }^{(18)}$. Hoy en día, la tomografía de arterias coronarias se ha ido posicionando como un estudio de utilidad puesto que permite calcular el diámetro, el sitio anatómico de drenaje, el espacio alrededor de la fístula y permite la planeación terapéutica ${ }^{(19,20)}$. Sin embargo, tal y como en el caso presentado, la mayoría de las fístulas coronarias son diagnosticadas durante una arteriografía coronaria realizada bajo otra sospecha diagnóstica. Esta técnica es el "gold estándar" que permite realizar una evaluación estructural y hemodinámica al igual que efectuar una intervención endovascular, en caso de considerarlo.

En general, para las fístulas coronarias asintomáticas se recomienda seguimiento y optimización del manejo médico para comorbilidades ${ }^{(21)}$. Se estima que el cierre espontáneo de las mismas ocurre en 1-2\% de los pacientes, especialmente en fístulas de tamaño pequeño, ausencia de comorbilidades cardiovasculares y con drenaje hacia el ventrículo derecho (21-23). Por el contrario, aquellas fístulas superior a $2 \mathrm{~cm}$ de diámetro o asociadas a complicaciones requieren el cierre de las mismas ${ }^{(21,24)}$.

El uso del cociente pulmonar/sistémico (Qp/Qs) permite cuantificar el grado de shunt y tomar decisiones clínicas ${ }^{(25)}$. Siendo 1 el valor aceptado como normal, se considera que una fístula coronaria con Qp/Qs $\geq 1,5$ debe ser valorado para su cierre, especialmente cuando hay historia de complicaciones o síntomas relacionados ${ }^{(26)}$

El método de elección (quirúrgico o endovascular) depende del juicio y experiencia del grupo de cardiología y el cirujano cardiovascular. El método percutáneo transcatéter tiene una tasa de éxito cercana al $90 \%$ y se prefiere en los casos de fístulas proximales, con único sitio de drenaje o con perfil de riesgo quirúrgico elevado ${ }^{(27,28)}$.

En los casos sintomáticos sin posibilidad de procedimiento de cierre, el uso de beta bloqueadores, bloqueadores de canales de calcio y antiagregantes plaquetarios han demostrado utilidad en el control de los síntomas ${ }^{(29)}$.

\section{Conclusiones}

La fístula coronaria es una anomalía rara que puede estar o no asociado a otras cardiopatías. Los síntomas más frecuentes reportados incluyen disnea y dolor torácico. Si bien la mayoría de los diagnósticos se realiza de forma incidental a través de la arteriografía coronaria. El tratamiento de esta entidad debe ser de forma integral por parte del equipo de cardiología en función de síntomas y tamaño del defecto.

\section{Bibliografía}

1- Raju MG, Goyal SK, Punnam SR, Shah DO, Smith GF, Abela GS. Coronary artery fistula: A case series with review of the literature. J Cardiol. 2009; 53(3):467-72.

2- Musante C, Giacomelli EM, Baliño PP. Fístula coronario-pulmonar. Rev Argent Cardiol. 2011;79(3):26768.

3- Gowda RM, Vasavada BC, Khan IA. Coronary artery fistulas: Clinical and therapeutic considerations. Int J Cardiol. 2006;107(1):7-10.

4- Abdelmoneim SS, Mookadam F, Moustafa SE, Holmes DR. Coronary Artery Fistula with Anomalous Coronary Artery Origin: A Case Report. J Am Soc Echocardiogr. 2007; 20(3):333.e1-333.e4. 
5- Majidi M, Shahzamani M, Mirhoseini M. Clinical features of coronary artery fistula. J Tehran Univ Hear Cent. 2011;5(3):158-61.

6- Durán A, Michelis V, Díaz P, Lujambio M, Kuster F, Lluberas R, et al. Evaluación de pacientes portadores de fístulas coronario-ventriculares múltiples. Rev méd Urug. 2003;19:237-41.

7- Hobbs RE, Millit HD, Raghavan P V., Moodie DS, Sheldon WC. Coronary artery fistulae: a 10-year review. Cleve Clin J Med. 1982;49(4):191-8.

8- Luo L, Kebede S, Wu S, Stouffer GA. Coronary artery fistulae. Am J Med Sci. 2006;332(2):79-84.

9- Sharma U, Aslam A, Tak T. Diagnosis of Coronary Artery Fistulas: Clinical Aspects and Brief Review of the Literature. Int J Angiol. 2013;22(03):189-92.

10- Sánchez R, Medina L, Cabrales J, Echeverri D. Fístula de arteria coronaria derecha a arteria pulmonar. Reporte de un caso. Rev Colomb Cardiol. 2010;17(5):214-6.

11- Fortunato Júnior JA, Branco Filho AA, Granzotto PCN, Moreira LMS, Martins ALM, Pereira ML, et al. Videotoracoscopia para fechamento de fístula coronário-pulmonar: Relato de caso. Rev Bras Cir Cardiovasc. 2010; 25(1):109-11.

12- Cotton JL. Diagnosis of a left coronary artery to right ventricular fistula with progression to spontaneous closure. J Am Soc Echocardiogr. 2000;13(3):225-8.

13- Qureshi SA. Coronary arterial fistulas. Orphanet J Rare Dis. 2006;1(1):51.

14- Verdini D, Vargas D, Kuo A, Ghoshhajra B, Kim P, Murillo H, et al. Coronary-Pulmonary Artery Fistulas. J Thorac Imaging. 2016;31(6):380-90.

15- Albeyoglu S, Aldag M, Ciloglu U, Sargin M, Oz TK, Kutlu H, et al. Coronary Arteriovenous Fistulas in Adult Patients: Surgical Management and Outcomes. Brazilian J Cardiovasc Surg. 2017;32(1):15-21.

16- Cárdenas L, Hidalgo F, López C, Paredes I, Cristhian ER. Isquemia miocárdica secundaria a fenómeno de robo de flujo por fístulas coronarias: reporte de caso. Rev Uruguaya Cardiol. 2019;34(2).

17- Rodríguez Silvestre Y, Alves do Couto A, Pessuti F, Weinert Menegaz M. Fístula entre la arteria coronaria descendente anterior y el tronco de la arteria pulmonar: Reporte de un caso clínico. Gac Médica Boliv. 2015;38(2):55-7.

18- Vitarelli A, De Curtis G, Conde Y, Colantonio M, Di Benedetto G, Pecce P, et al. Assessment of congenital coronary artery fistulas by transesophageal color Doppler echocardiography. Am J Med. 2002;113(2):127-33.

19- Candan Ö. Imaging of a large coronary fistula using by echocardiography. Turk Kardiyol Dern ArsiviArchives Turkish Soc Cardiol. 2018;46(6):507-9.

20- Punzo B, Forte E, Salvatore M, Cavaliere C, Cademartiri F. Case report of coronary artery fistula. Medicine (Baltimore). 2019;98(49):e18255.

21- Buccheri D, Chirco PR, Geraci S, Caramanno G, Cortese B. Coronary Artery Fistulae: Anatomy, Diagnosis and Management Strategies. Hear Lung Circ. 2018;27(8):940-51.

22- Jaffe RB, Glancy DL, Epstein SE, Brown BG, Morrow AG. Coronary Arterial-Right Heart Fistulae. Circulation. 1973;47(1):133-43.

23- Santoro G, Formigari R, Di Carlo D, Ballerini L. Spontaneous closure of coronary fistulas: a possible prognostic role of the site of drainage. G Ital Cardiol. 1994;24(10):1223-6.

24- Stout KK, Daniels CJ, Aboulhosn JA, Bozkurt B, Broberg CS, Colman JM, et al. 2018 AHA/ACC Guideline for the Management of Adults With Congenital Heart Disease: A Report of the American College of Cardiology/American Heart Association Task Force on Clinical Practice Guidelines. Circulation. 2019; 139(14):e637-97.

25- Marín Rodríguez C, Sánchez Alegre ML, Lancharro Zapata Á, Alarcón Rodríguez J. Cuantificación de la circulación pulmonar y sistémica: $Q p / Q s$. Qué es, cómo se calcula y para qué sirve. Lo que el radiólogo debe saber. Radiologia. 2015;57(5):369-79.

26- Lara de la Fuente I, Estrada Parra I, Villagómez Villegas D, Pastor Torres L. Fístula coronaria como causa de insuficiencia cardíaca. Cardiocore. 2013;48(3):124-6.

27- Challoumas D, Pericleous A, Dimitrakaki I, Danelatos C, Dimitrakakis G. Coronary Arteriovenous Fistulae: A Review. Int J Angiol. 2014;23(01):001-10. 
28- Liu X, Zhang L, Qi Z, Fan M, Ge J. The characteristics of coronary-pulmonary artery fistulas and the effectivity of trans-catheter closure: a single center experience. J Thorac Dis. 2019;11(7):2808-15.

29- Kim H, Beck KS, Choe YH, Jung Jl. Coronary-to-Pulmonary Artery Fistula in Adults: Natural History and Management Strategies. Korean J Radiol. 2019;20(11):1491.

\section{Aporte cada autor al trabajo}

Jenny Carolina Salazar F: concepción y diseño del trabajo, análisis e interpretación de los datos, redacción del manuscrito.

Diego Alejandro Rangel R: concepción y diseño del trabajo, recolección de datos, redacción del manuscrito.

Néstor Eduardo Ospino R: concepción y diseño del trabajo, recolección de datos, revisión crítica del manuscrito.

Juan Manuel Quirós: concepción y diseño del trabajo, recolección de datos, revisión crítica del manuscrito. 